\title{
Changing Proportions of HIV-1 Subtypes and Transmitted Drug Resistance Among Newly Diagnosed HIV/AIDS Individuals - China, 2015 and 2018
}

\author{
Jingjing Hao'; Shan Zheng'; Mengze Gan'; Aobo Dong'; Ruihua Kang'; Miaomiao Li'; Shuai Zhao'; \\ Jing $\mathrm{Hu}^{1}$; Chang Song'; Lingjie Liao'; Yi Feng'; Yiming Shao'; Yuhua Ruan'; Hui Xing ${ }^{1}{ }^{1, *}$
}

\begin{abstract}
Introduction: With the expansion of human immunodeficiency virus (HIV) antiretroviral therapy (ART), HIV drug resistance is becoming more and more serious. This study describes the changing prevalence of HIV-1 subtypes and transmitted drug resistance (TDR) among newly diagnosed individuals in China, 2015 and 2018.

Methods: A total of 8,980 individuals in 2015 and 2018 from 31 provincial-level administrative divisions (PLADs) were enrolled in this study. Viral RNAs were amplified and sequenced using an in-house polymerase chain reaction (PCR) protocol. The Stanford HIV Drug Resistance Database (HIVdb) was used to predict susceptibility to 12 antiretroviral drugs.
\end{abstract}

Results: The prevalence of TDR was not significantly increased over time. The prevalence of TDR was $3.8 \%$ and $4.4 \%$ in 2015 and 2018, respectively $(P=0.13)$. The prevalence of CRF55_01B increased from $2.3 \%$ in 2015 to $3.9 \%$ in 2018 $(P<0.001)$. The drug resistance prevalence of nonnucleoside reverse transcriptase inhibitors (NNRTI) increased from $2.4 \%$ in 2015 to $3.3 \%$ in 2018 $(P<0.01)$. The prevalence of E138 $(P<0.001), \mathrm{H} 221$ $(P=0.03)$, and V179 $(P<0.001)$ mutations increased from $0.30 \%, 0.09 \%$, and $0.70 \%$ in 2015 to $1.10 \%$, $0.30 \%$, and $1.70 \%$ in 2018 , respectively.

Conclusions: HIV drug resistance affects the effect of antiretroviral treatment, so the monitoring of HIV TDR should be strengthened to control the transmission of HIV drug resistance.

\section{INTRODUCTION}

The four main human immunodeficiency virus (HIV)-1 subtypes in China over the past 20 years include CRF07_BC, CRF01_AE, CRF08_BC, and subtype $\mathrm{B}$. The prevalence of CRF07_BC and
CRF01_AE has increased since 2006 and they remain the dominant subtypes in China present in 31 provincial-level administrative divisions (PLADs) (1). In 2012, a study reported the HIV-1 CRF55_01B subtype, composed of CRF01_AE and subtype B, which possibly originated in men having sex with men (MSM) in Shenzhen (2). After its origin in Shenzhen, it spread rapidly to all cities in China (3). Various factors contribute to the spread and diversification of HIV-1 subtypes in China, including the emergence of more dating apps, the rapid development of the economy and means of transportation, and people moving to different parts of the country for employment.

From 2012, several first-line antiretroviral drugs were available to HIV patients for free, including TDF/AZT+3TC+EFV/NVP. In 2014, only HIV patients with a cluster of differentiation 4 (CD4) cell count of less than 500 cells $/ \mu \mathrm{L}$ were eligible to receive ART. The Chinese government adjusted the standard for free ART in 2016 and recommended that all HIV patients receive ART (4). Adherence to ART is critical to achieving viral suppression; however, poor drug adherence or withdrawal from treatment can result in the development of drug resistance (5). Moreover, the problem of drug resistance can be exacerbated by the expansion of ART regimens. One possible solution would be conducting TDR surveys, which can offer an effective guide for structuring future first- and secondline ART regimens.

\section{METHODS}

The study design was conducted according to the WHO protocol for TDR in each region of China. Depending on the number of newly reported HIV/AIDS cases, China was divided into high prevalence regions, moderate prevalence regions and low prevalence regions. At least 300 patients were recruited in high prevalence regions, moderate 
prevalence regions and low prevalence regions $(6-7)$. The individuals (16 years old or older at the time of HIV-1 diagnosis) diagnosed with HIV-1 infection in 2015 and 2018 were enrolled in this study. All individuals provided written informed consent. Study variables included the following epidemiological and clinical characteristics: age, sex, education, HIV-1 transmission route, and HIV-1 subtype. Plasma from participants was collected and sent to the laboratory via cold chain transport.

Viral RNAs were extracted from $200 \mu \mathrm{L}$ of plasma using the QIAsymphony platform. The pol fragment (HXB2 positions 2,253-3,312, 1,060 bp) was amplified and sequenced using an in-house PCR protocol. The nucleotide sequences were aligned separately using the HIV Align tool (https://mafft. cbrc.jp/alignment/server/add_fragments.html). The aligned sequences were manually adjusted using BioEdit (version 7.0.9.1, Borland, CA, USA). Sequences less than 1,000 nucleotides in length were excluded from the analysis. HIV-1 subtypes were determined based on a neighbor-joining tree with Mega [Mega 7.0: Molecular Evolutionary Genetics Analysis across computing platforms (Kumar S, Stecher G, and Tamura K 2016)]. The Stanford HIV Drug Resistance Database (HIVdb) (https://HIV$1 \mathrm{db}$.stanford.edu/HIV-1db/by-sequences) genotypic resistance interpretation system was used to predict susceptibility to 12 antiretroviral drugs recommended for use by the WHO (EFV, NVP, ABC, AZT, 3TC, TDF, FTC, D4T, DDI, LPV/r, ATV/r, and DRV/r). This system assigns a drug penalty score to each mutation. Scores for individual mutations are greater than -15: negative numbers indicate increased susceptibility compared to wild type virus; 0 indicates no change to susceptibility; susceptible (0-9), potential low-level resistance (10-14), low-level resistance (15-29), intermediate resistance (30-59), and highlevel resistance $(\geq 60)$. Drug resistance is defined as 15 points or more.

SAS (version 9.4, SAS Institute Inc., Cary, NC, USA) was used for statistical analysis. The $\chi^{2}$ test was used to analyze variations of 12 types of drugs and drug resistance mutations in 2015 and 2018. Univariate and multivariate logistic regression tests were used to analyze the factors associated with drug resistance, with $P<0.05$ considered as statistically significant.

\section{RESULTS}

A total of 8,980 individuals were diagnosed with
HIV-1 in 2015 and 2018, with 4,704 diagnosed in 2015 and 4,276 in 2018. The characteristics of the individuals included in this study are listed in Table 1. The proportions of HIV-1 individuals aged 26-49 and $\geq 50$ years old were $56.8 \%$ and $24.8 \%$, respectively. Approximately $81.9 \%$ of HIV-1 individuals were men. About $40.6 \%$ of HIV-1 individuals graduated from high school or had higher education. The main pattern of transmission was heterosexual intercourse $(52.5 \%)$.

The most common HIV-1 subtype was CRF07_BC (39.3\%), followed by CRF01_AE (36.2\%), CRF08_BC (8.9\%), B (4.3\%), and CRF55_01B (3.1\%) (Table 1). The prevalence of CRF55_01B increased from $2.3 \%$ in 2015 to $3.9 \%$ in 2018 $(P<0.001)$ (Table 2).

The TDR prevalence was $3.80 \%$ in 2015 and $4.40 \%$ in 2018; overall TDR prevalence did not change significantly between 2015 and 2018. TDR prevalence of NNRTI, NRTI, and PI changed from $2.40 \%$, $1.10 \%$, and $0.20 \%$ in 2015 , to $3.30 \%, 0.80 \%$, and $0.07 \%$ in 2018 , respectively. The increased prevalence of NNRTI drug resistance was from $2.40 \%$ in 2015 to $3.30 \%$ in $2018(P<0.01)$, whereas no increase in drug resistance was observed for NRTI and PI. Moreover, the prevalence of certain mutations, including E138, $\mathrm{H} 221$, and V179, significantly increased from $0.30 \%$, $0.09 \%$, and $0.70 \%$ in 2015 to $1.10 \%, 0.30 \%$, and $1.70 \%$ in 2018 , respectively (Table 3 ).

Multivariate logistic regression analysis showed that CRF08_BC [adjusted odds ratio $(\mathrm{AOR})=1.51,95 \%$ confidence interval (CI): 1.03-2.23], CRF55_01B $(\mathrm{AOR}=4.17, \quad 95 \% \quad \mathrm{CI}: 2.77-6.27)$, and URF $(\mathrm{AOR}=1.69,95 \% \mathrm{CI}: 1.14-2.49)$ were independent factors associated with TDR (Table 4).

\section{DISCUSSION}

In China, CRF07_BC and CRF01_AE were still the two main subtypes. The proportion of the CRF55_01B subtype increased from 2015 to 2018. As a new recombinant subtype formed by the recombination of CRF01_AE and subtype $B$, CRF55_01B prevalence increased exponentially from 2005 to 2009 after its origin in Shenzhen. One of the factors contributing to its rapid transmission was the spread of the virus to different PLADs in 2007, as mediated by the rapid development of the BeijingGuangzhou and Beijing-Kowloon railways (8). Moreover, even though this particular subtype 
TABLE 1. General characteristics of newly diagnosed HIV individuals in 2015 and 2018, China.

\begin{tabular}{|c|c|c|c|c|c|c|}
\hline \multirow{3}{*}{$\begin{array}{ll} & \text { Variable } \\
\text { Total }\end{array}$} & \multicolumn{2}{|c|}{2015} & \multicolumn{2}{|c|}{2018} & \multicolumn{2}{|c|}{ Total } \\
\hline & \multicolumn{2}{|c|}{ Number of cases Proportion (\%) } & \multicolumn{2}{|c|}{ Number of cases Proportion (\%) } & \multicolumn{2}{|c|}{ Number of cases Proportion (\%) } \\
\hline & 4,704 & 100.0 & 4,276 & 100.0 & 8,980 & 100.0 \\
\hline \multicolumn{7}{|l|}{ Age (years) } \\
\hline $16-25$ & 862 & 18.3 & 676 & 15.8 & 1,538 & 17.1 \\
\hline $26-49$ & 2,749 & 58.5 & 2,350 & 55.0 & 5,099 & 56.8 \\
\hline$\geq 50$ & 1,003 & 21.3 & 1,227 & 28.7 & 2,230 & 24.8 \\
\hline Unknown & 90 & 1.9 & 23 & 0.5 & 113 & 1.3 \\
\hline \multicolumn{7}{|l|}{ Sex } \\
\hline Male & 3,763 & 80.0 & 3,595 & 84.1 & 7,358 & 81.9 \\
\hline Female & 876 & 18.6 & 681 & 15.9 & 1,557 & 17.4 \\
\hline Unknown & 65 & 1.4 & 0 & 0.0 & 65 & 0.7 \\
\hline \multicolumn{7}{|l|}{ Education } \\
\hline Primary school & 1,338 & 28.5 & 993 & 23.2 & 2,331 & 26.0 \\
\hline Junior high school & 1,412 & 30.0 & 1,205 & 28.2 & 2,617 & 29.1 \\
\hline Senior high school or above & 1,769 & 37.6 & 1,877 & 43.9 & 3,646 & 40.6 \\
\hline Unknown & 185 & 3.9 & 201 & 4.7 & 386 & 4.3 \\
\hline \multicolumn{7}{|l|}{ Route of HIV transmission } \\
\hline HET & 2,533 & 53.9 & 2,181 & 51.0 & 4,714 & 52.5 \\
\hline MSM & 1,855 & 39.4 & 1,906 & 44.6 & 3,761 & 41.9 \\
\hline IDU & 115 & 2.4 & 53 & 1.2 & 168 & 1.9 \\
\hline Others & 201 & 4.3 & 136 & 3.2 & 337 & 3.7 \\
\hline \multicolumn{7}{|l|}{ Subtype } \\
\hline CRF01_AE & 1,835 & 39.0 & 1,578 & 36.9 & 3,413 & 38.0 \\
\hline CRF07_BC & 1,675 & 35.6 & 1,696 & 39.7 & 3,371 & 37.5 \\
\hline CRF08_BC & 418 & 8.9 & 384 & 9.0 & 802 & 8.9 \\
\hline CRF55_01B & 110 & 2.3 & 168 & 3.9 & 278 & 3.1 \\
\hline B & 261 & 5.6 & 183 & 4.3 & 444 & 5.0 \\
\hline Others & 405 & 8.6 & 267 & 6.2 & 672 & 7.5 \\
\hline
\end{tabular}

Abbreviations: HIV=human immunodeficiency virus; HET=heterosexual; MSM=men who have sex with men; IDU=injecting drug users.

TABLE 2. Changes in HIV subtypes among newly diagnosed HIV individuals in 2015 and 2018, China.

\begin{tabular}{|c|c|c|c|c|c|}
\hline \multirow{2}{*}{ Subtype } & \multicolumn{2}{|c|}{2015} & \multicolumn{2}{|c|}{2018} & \multirow{2}{*}{$P$} \\
\hline & Number of cases & Proportion (\%) & Number of cases & Proportion (\%) & \\
\hline CRF01_AE & 1,835 & 39.0 & 1,578 & 36.9 & 0.04 \\
\hline CRF07_BC & 1,675 & 35.6 & 1,696 & 39.7 & $<0.0001$ \\
\hline CRF08_BC & 418 & 8.9 & 384 & 9.0 & 0.88 \\
\hline CRF55_01B & 110 & 2.3 & 168 & 3.9 & $<0.0001$ \\
\hline B & 261 & 5.6 & 183 & 4.3 & 0.006 \\
\hline Others & 405 & 8.6 & 267 & 6.2 & $<0.0001$ \\
\hline
\end{tabular}

Abbreviation: HIV=human immunodeficiency virus.

originated in homosexual males, it was transmitted among heterosexuals. Currently, in-depth studies on CRF55_01B are lacking, especially as it relates to the underlying mechanism of drug resistance.

The TDR prevalence was $3.80 \%$ in 2015 and $4.40 \%$ in 2018, with no significant increase over time. A study 
TABLE 3. Changes of HIV mutations among newly diagnosed HIV individuals in 2015 and 2018, China.

\begin{tabular}{|c|c|c|c|c|c|}
\hline \multirow[b]{2}{*}{ Variable } & \multicolumn{2}{|c|}{2015} & \multicolumn{2}{|c|}{2018} & \multirow[b]{2}{*}{$P$} \\
\hline & $\begin{array}{l}\text { Number } F \\
\text { of cases }\end{array}$ & $\begin{array}{c}\text { roportion } \\
(\%)\end{array}$ & $\begin{array}{l}\text { Number } \\
\text { of cases }\end{array}$ & $\begin{array}{c}\text { oportion } \\
(\%)\end{array}$ & \\
\hline Total & 178 & 3.80 & 189 & 4.40 & 0.13 \\
\hline NNRTI & 113 & 2.40 & 142 & 3.30 & 0.01 \\
\hline A98G & 5 & 0.10 & 7 & 0.20 & 0.46 \\
\hline $\mathrm{E} 138 \mathrm{~A} / \mathrm{G} / \mathrm{K} / \mathrm{Q}$ & 13 & 0.30 & 45 & 1.10 & $<0.001$ \\
\hline G190A & 13 & 0.30 & 9 & 0.20 & 0.53 \\
\hline $\mathrm{H} 221 \mathrm{Y}$ & 4 & 0.09 & 12 & 0.30 & 0.03 \\
\hline K101E & 15 & 0.30 & 11 & 0.30 & 0.59 \\
\hline K103N/S & 34 & 0.70 & 34 & 0.80 & 0.69 \\
\hline L100I & 1 & 0.02 & 1 & 0.02 & 1.00 \\
\hline $\mathrm{P} 225 \mathrm{H}$ & 1 & 0.02 & 5 & 0.10 & 0.11 \\
\hline V106I/M & 16 & 0.30 & 23 & 0.50 & 0.15 \\
\hline V108I & 15 & 0.30 & 8 & 0.20 & 0.22 \\
\hline V179A/D & 35 & 0.70 & 72 & 1.70 & $<0.001$ \\
\hline Y181C & 7 & 0.20 & 11 & 0.30 & 0.25 \\
\hline NRTI & 52 & 1.10 & 33 & 0.80 & 0.10 \\
\hline D67N & 3 & 0.06 & 4 & 0.09 & 0.72 \\
\hline K219Q & 3 & 0.06 & 2 & 0.05 & 1.00 \\
\hline K65R & 7 & 0.10 & 7 & 0.20 & 0.86 \\
\hline K70E/R & 8 & 0.20 & 8 & 0.20 & 0.85 \\
\hline L210W & 7 & 0.20 & 3 & 0.07 & 0.35 \\
\hline L74I & 2 & 0.04 & 2 & 0.05 & 1.00 \\
\hline M184I/V & 15 & 0.30 & 9 & 0.20 & 0.32 \\
\hline M41L & 9 & 0.20 & 5 & 0.10 & 0.37 \\
\hline T69D/N & 6 & 0.10 & 0 & 0.00 & 0.03 \\
\hline V75M/A & 2 & 0.04 & 2 & 0.05 & 1.00 \\
\hline Y115F & 1 & 0.02 & 1 & 0.02 & 1.00 \\
\hline $\mathrm{PI}$ & 7 & 0.20 & 3 & 0.07 & 0.26 \\
\hline I50V/L & 1 & 0.02 & 1 & 0.02 & 1.00 \\
\hline I54L/M & 1 & 0.02 & 0 & 0.00 & 1.00 \\
\hline M46I/L & 3 & 0.06 & 2 & 0.05 & 1.00 \\
\hline F53L & 1 & 0.02 & 0 & 0.00 & 1.00 \\
\hline $147 \mathrm{~V}$ & 2 & 0.04 & 0 & 0.00 & 1.00 \\
\hline L90M & 0 & 0.00 & 1 & 0.02 & 0.96 \\
\hline L33F & 3 & 0.06 & 1 & 0.02 & 0.63 \\
\hline
\end{tabular}

Abbreviations: HIV=human immunodeficiency virus; NNRTI=nonnucleoside reverse transcriptase inhibitor; NRTI=nucleoside reverse transcriptase inhibitor; $\mathrm{Pl}=$ protease inhibitor.

that included TDR prevalence data from 2001 to 2017 in China estimated that TDR prevalence was $4.1 \%$ (9), which is consistent with the results of this study. Reports on drug resistance testing from the WHO revealed that between 2014 and 2019 , the median rate of transmission-resistant drugs was $4.1 \%$ in Southeast Asia, $6.0 \%$ in Sub-Saharan Africa, $9.1 \%$ in Latin America, $8.5 \%$ in Europe, and $14.2 \%$ in North America (10). Conversely, the prevalence of TDR was much higher in Europe and North America, which was consistent with the longer existence and variety of antiviral treatment.

The prevalence of transmitted NNRTI resistance increased from 2015 to 2018 . According to the analysis of resistance mutations, the prevalence of the E138, $\mathrm{H} 221$, and V179 mutations increased. V179E is a nonpolymorphic mutation weakly selected by NVP and EFV, E138G is another nonpolymorphic accessory mutation occasionally in patients receiving NVP and EFV (11). Molecular monitoring results of newly diagnosed individuals in China showed that the most common mutations in patients' resistance to NNRTI were E138G and V179E (12). Moreover, E138K was found to be the most common mutation in HIV-1 patients resistant to NNRTI in the US, according to an analysis of TDR from 2014 to 2018 (10). The impact of the combination of E138 and V179 on both NNRTI susceptibility and virologic outcome in patients deserves investigation.

CRF55_01B was associated with drug resistance. Moreover, V179E often appears in CRF55_01B together with E138G and has low resistance to EFV and NVP (13). The rapid spread of the CRF55_01B subtype across China was mediated by an increase of plasma HIV RNA load and the relatively lower CD4 count (14). Moreover, these factors may prolong the asymptomatic phase and increase the risk of HIV transmission, which may soon lead to further increases and a potential epidemic. Finally, these factors may also help explain the recent surging dominance and continued expansion of this subtype among MSM in China.

This study was subject to several limitations. First, during the onsite questionnaire survey, the information was not filled in detail, such as ID number, so there were deviations in the data analysis. The second is that the two-year sequences were obtained by the same method, but not by the same team, so there will be certain differences in comparison. The experimental methods were the same, but the Stanford drug resistance discriminant version was different. This study retested for resistance against the latest version.

Although the overall prevalence of TDR was low in China, it is necessary to remain vigilant, especially of 
TABLE 4. Factors associated with HIV drug resistance among newly diagnosed HIV individuals in 2015 and 2018, China.

\begin{tabular}{|c|c|c|c|c|c|c|}
\hline Factors & Total & TDR (\%) & OR $(95 \% \mathrm{Cl})$ & $P$ & AOR $(95 \% \mathrm{Cl})$ & $P$ \\
\hline Total & 8,980 & $367(4.1)$ & & & & \\
\hline \multicolumn{7}{|l|}{ Age (years) } \\
\hline $16-25$ & 2,230 & $78(3.5)$ & 1.00 & & 1.00 & \\
\hline $26-49$ & 5,099 & $209(4.1)$ & $1.24(0.95-1.61)$ & 0.12 & $1.21(0.93-1.58)$ & 0.16 \\
\hline$\geq 50$ & 1,538 & $72(4.7)$ & $1.41(1.02-1.95)$ & 0.04 & $1.39(1.00-1.94)$ & 0.05 \\
\hline Unknown & 113 & $8(7.1)$ & $2.11(1.00-4.48)$ & 0.05 & $2.05(0.96-4.37)$ & 0.06 \\
\hline \multicolumn{7}{|l|}{ Sex } \\
\hline Male & 7,358 & $299(4.1)$ & 1.00 & & & \\
\hline Female & 1,557 & $65(4.2)$ & $1.03(0.78-1.35)$ & 0.84 & & \\
\hline Unknown & 65 & $3(4.6)$ & $1.12(0.35-3.60)$ & 0.84 & & \\
\hline \multicolumn{7}{|l|}{ Education } \\
\hline Primary school & 2,331 & $90(3.9)$ & 1.00 & & & \\
\hline Junior high school & 2,617 & $120(4.6)$ & $1.20(0.91-1.58)$ & 0.21 & & \\
\hline Senior high school or above & 3,646 & $147(4.0)$ & $1.05(0.80-1.37)$ & 0.74 & & \\
\hline Unknown & 386 & $10(2.6)$ & $0.66(0.34-1.28)$ & 0.22 & & \\
\hline \multicolumn{7}{|l|}{ Route of HIV transmission } \\
\hline HET & 4,714 & $201(4.3)$ & 1.00 & & & \\
\hline MSM & 3,761 & $140(3.7)$ & $0.87(0.70-1.08)$ & 0.21 & & \\
\hline IDU & 168 & $11(6.5)$ & $1.57(0.84-2.95)$ & 0.16 & & \\
\hline Others & 337 & $15(4.5)$ & $1.04(0.61-1.78)$ & 0.89 & & \\
\hline \multicolumn{7}{|l|}{ Subtype } \\
\hline CRF01_AE & 3,413 & $135(4.0)$ & 1.00 & & 1.00 & \\
\hline CRF07_BC & 3,371 & $107(3.2)$ & $1.26(0.97-1.63)$ & 0.08 & $1.25(0.97-1.62)$ & 0.09 \\
\hline CRF08_BC & 802 & $37(4.6)$ & $1.48(1.01-2.16)$ & 0.05 & $1.51(1.03-2.23)$ & 0.04 \\
\hline CRF55_01B & 278 & $34(12.2)$ & $4.25(2.83-6.39)$ & $<0.001$ & $4.17(2.77-6.27)$ & $<0.001$ \\
\hline B & 444 & $19(4.3)$ & $1.36(0.83-2.25)$ & 0.22 & $1.36(0.83-2.24)$ & 0.23 \\
\hline Others & 672 & $35(5.2)$ & $1.67(1.13-2.47)$ & 0.01 & $1.69(1.14-2.49)$ & 0.01 \\
\hline \multicolumn{7}{|l|}{ Year } \\
\hline 2015 & 4,704 & $178(3.8)$ & 1.00 & & & \\
\hline 2018 & 4,276 & $189(4.4)$ & $1.18(0.95-1.45)$ & 0.13 & & \\
\hline
\end{tabular}

Abbreviations: HIV=human immunodeficiency virus; TDR=transmitted drug resistance; OR=odds ratio; $\mathrm{Cl}=$ confidence interval; $A O R=$ adjusted odds ratio; $H E T=$ heterosexual; $M S M=$ men who have sex with men; IDU=injecting drug users.

CRF55_01B. Strengthened surveillance of TDR can help reduce the transmission of TDR to the key target populations, especially MSM. In 2018, TDR reached a moderate epidemic level in Xinjiang Uygur Autonomous Region and other regions. Key areas should pay attention and take measures to control the spread of drug resistance. Reducing the prevalence of TDR is important for formulating future treatment and prevention guidelines. Surveillance and prevention of drug resistance should be a critical component of China's programmatic response to HIV to ensure the long-term efficacy and sustainability of ART and achieve 95-95-95 targets.

Conflicts of interest: No conflicts of interest.

Acknowledgments: Provincial CDC staff.

Funding: The Ministry of Science and Technology of China (2017ZX10201101).

doi: $10.46234 / \mathrm{ccdcw} 2021.251$

\# Corresponding author: Xing Hui, xingh@chinaaids.cn.

\footnotetext{
${ }^{1}$ State Key Laboratory of Infectious Disease Prevention and Control (SKLID), National Center for AIDS/STD Control and Prevention (NCAIDS), Chinese Center for Disease Control and Prevention (China CDC), Collaborative Innovation Center for Diagnosis and Treatment of Infectious Diseases, Beijing, China.
} 
Submitted: October 22, 2021; Accepted: November 19, 2021

\section{REFERENCES}

1. He X, Xing $\mathrm{H}$, Ruan $\mathrm{YH}$, Hong KX, Cheng CL, Hu YY, et al. A comprehensive mapping of HIV-1 genotypes in various risk groups and regions across China based on a nationwide molecular epidemiologic survey. PLoS One 2012;7(10):e47289. http://dx.doi.org/10.1371/ journal.pone.0047289.

2. Han XX, An MH, Zhang WQ, Cai WP, Chen X, Takebe Y, et al. Genome sequences of a novel HIV-1 circulating recombinant form, CRF55_01B, identified in China. Genome Announc 2013;1(1):e00050 - 12. http://dx.doi.org/10.1128/genomeA.00050-12.

3. Liang BY, Wei QY, Yang Y, Yang Y, Liu J, Chu JM, et al. Identification of a novel HIV-1 CRF55_01B/B recombinant isolate in Guangxi, China. AIDS Res Hum Retroviruses 2020;36(5):434 - 9. http://dx.doi. org/10.1089/aid.2019.0222.

4. Chinese Center for Disease Control and Prevention. Notice of the General Office of the National Health and Family Planning Commission on adjusting the standard of free antiviral treatment for AIDS. 2016. http://www.chinaaids.cn/zlgh/hdjz6/201606/t20160615_ 131433.htm. [2016-6-15]. (In Chinese).

5. Benson C, Wang X, Dunn KJ, Li N, Mesana L, Lai J, et al. Antiretroviral adherence, drug resistance, and the impact of social determinants of health in HIV-1 patients in the US. AIDS Behav 2020;24(12):3562 - 73. http://dx.doi.org/10.1007/s10461-020-02937-

6. Kang RH, Liang SJ, Ma YL, Liang S, Xiao L, Zhang XH, et al. Pretreatment HIV drug resistance in adults initiating antiretroviral therapy in China, 2017. Infect Dis Poverty 2020;9(1):54. http://dx.doi. org/10.1186/s40249-020-00668-5.
7. World Health Organization. HIV drug resistance surveillance guidance, 2015 update. 2016. https://apps.who.int/iris/handle/10665/204471. [2020-2-5].

8. Gan MZ, Zheng S, Hao JJ, Ruan YH, Liao LJ, Shao YM, et al. The prevalence of CRF55_01B among HIV-1 strain and its connection with traffic development in China. Emerg Microbes Infect 2021;10(1):256 65. http://dx.doi.org/10.1080/22221751.2021.1884004

9. Ye JR, Hao MQ, Xing H, Zhang FJ, Wu H, Lv W, et al. Transmitted HIV drug resistance among individuals with newly diagnosed HIV infection: a multicenter observational study. AIDS 2020;34(4):609 19. http://dx.doi.org/10.1097/QAD.0000000000002468.

10. McClung RP, Oster AM, Ocfemia MCB, Saduvala N, Heneine W, Johnson JA, et al. Transmitted drug resistance among HIV-1 diagnoses in the United States, 2014-2018. Clin Infect Dis, 2021ciab583. http:// dx.doi.org/10.1093/cid/ciab583.

11. Liu YJ, Li HP, Wang XL, Han JW, Jia L, Li TY, et al. Natural presence of $\mathrm{V} 179 \mathrm{E}$ and rising prevalence of E138G in HIV-1 reverse transcriptase in CRF55_01B viruses. Infect Genet Evol 2020;77: 104098. http://dx.doi.org/10.1016/j.meegid.2019.104098.

12. Zhang D, Zheng CL, Li HP, Li H, Liu YJ, Wang XL, et al. Molecular surveillance of HIV-1 newly diagnosed infections in Shenzhen, China from 2011 to 2018. J Infect 2021;83(1):76 - 83. http://dx.doi.org/10. 1016/j.jinf.2021.04.021.

13. Dong AB, Liu L, Xiao L, Liang S, Li K, Hu J, et al. First detection of a circulating recombinant form of HIV-1 CRF01_AE/08_BC (CRF105_0108) with drug-resistant mutations in Sichuan, China. AIDS Res Hum Retroviruses 2020;36(7):625 - 30. http://dx.doi.org/ 10.1089/AID.2020.0034.

14. Wei L, Li H, Lv X, Zheng CL, Li GL, Yang ZR, et al. Impact of HIV-1 CRF55_01B infection on the evolution of CD4 count and plasma HIV RNA load in men who have sex with men prior to antiretroviral therapy. Retrovirology 2021;18(1):22. http://dx.doi.org/10.1186/ s12977-021-00567-z. 